Original Article

\title{
Correlation study of knee joint proprioception test results using common test methods
}

\author{
Lin Li ${ }^{1)}$, ZhONG-QIU JI ${ }^{1{ }^{*}}$, YAN-XIA Li ${ }^{2)}$, WeI-TONG LiU' ${ }^{1)}$ \\ 1) College of Physical Education and Sports, Beijing Normal University: No. 19, XinJieKouWai St., \\ HaiDian District, Beijing 100875, China \\ 2) College of Physical Education, Langfang Teachers University, China
}

\begin{abstract}
Purpose] To study the correlation of the results obtained from different proprioception test methods, namely, the joint angle reset method, the motion minimum threshold measurement method, and the force sense reproduction method, performed on the same subjects' knees. [Subjects and Methods] Different proprioception test methods, the joint angle reset method, the motion minimum threshold measurement method and the force sense reproduction method were used to test the knees of 30 healthy young men. [Results] Correlations were found in the following descending order from strong to weak: the correlation between the joint angle reset method and the force sense reproduction method (correlation coefficient of 0.41 ), the correlation between the joint angle reset method and the motion minimum threshold measurement method (correlation coefficient of 0.29 ), the correlation between the motion minimum threshold measurement method and the force sense reproduce method (correlation coefficient of 0.15). [Conclusion] No correlation was found among the results obtained using the joint angle reset method, the motion minimum threshold measurement method and the force sense reproduction method. Therefore, no correlation was found among the position sense, the motion sense and the force sense represented by these methods. Using the results of only one of the test methods to represent proprioception is one-sided. Force sensation depends more on the sensory input of information from the Golgi tendon organs, motion sense depends more on the input information of the muscle spindles, and position sense relies on the double input information of the muscle spindles and the Golgi tendon organs.

Key words: Proprioception, Common test methods, Knee joint
\end{abstract}

(This article was submitted Sep. 16, 2015, and was accepted Oct. 31, 2015)

\section{INTRODUCTION}

Since 1906, when Charles Sherrington put forward the term proprioception ${ }^{1)}$, people both in China and overseas have conducted extensive research on this topic. This research includes the sensory receptors of proprioception, neural pathways, the nerve centres, test methods, training methods, influencing factors and other aspects ${ }^{2,3)}$. Many research achievements have been applied to the improvement of sports skills, the prevention and rehabilitation of sports injuries,and the risk assessment and prevention of falls by the elderly ${ }^{4,5}$.

The determination of proprioceptive ability is important for assessing the function of the joints. The test methods of proprioception currently include the joint angle reset method, the motion minimum threshold measurement method, the force sense reproduction method, the digital opposition test, the touch nose test, the contralateral matching test, the visual modelling method, the weight matching test, the dynamic and static balance tests, and many other methods. The most commonly used methods are the joint angle reset method, the motion minimum threshold measurement method, and the force sense reproduction method. Currently, the test results of only one test method are commonly used to measure the sensitivity of the

*Corresponding author. Zhong-Qiu Ji (E-mail: jizhongqiu61@bnu.edu.cn)

(C2016 The Society of Physical Therapy Science. Published by IPEC Inc.

This is an open-access article distributed under the terms of the Creative Commons Attribution Non-Commercial No Derivatives (by-nc-nd) License $<$ http://creativecommons.org/licenses/by-nc-nd/4.0/>. 
proprioception ${ }^{6}$. According to previous studies, different proprioception test methods used to examine the same subjects will often give different results ${ }^{7}$. For instance, Barrett and others used the joint angle retest method and concluded that proprioception was significantly improved after anterior cruciate ligament reconstruction surgery ${ }^{8}$. However, MacDonald and others used the motion minimum threshold measurement method as the test, and concluded that proprioception did not change after anterior cruciate ligament reconstruction surgery ${ }^{9,10)}$.

The objective of this research was to study the correlation of the results obtained from different proprioception test methods, namely, the joint angle reset method, the motion minimum threshold measurement method, and the force sense reproduction method, performed on the same subjects' knees.

\section{SUBJECTS AND METHODS}

Thirty male students were randomly recruited at Beijing Normal University as the experimental subjects. Table 1 shows the characteristics of the subjects. All of the subjects were required to be free of organic disease in the major organs and motor systems, and to have normal vision and normal joint range of motion of the knee joints on the dominant side.

This study complied with the ethical principles of the Declaration of Helsinki (1975, revised 1983). The study protocol was approved by Beijing Normal University Ethics Committee and written informed consent was obtained from each subject.

All of the tests were conducted in the Sports Biomechanics Laboratory at the College of Sport and Physical Education at Beijing Normal University. Only the subjects dominant side knee joints were tested, and the dominant side was determined by conducting a ball-kicking test. An isokinetic dynamometer device (BIODEX multi-joint muscle strength test system, System 4; Biodex Medical Systems, Inc., Shirley, NY, USA) was used to perform tests using the joint angle reset method, the motion minimum threshold measurement method and the force sense reproduction method.

The joint angle reset test: The subject sat on the testing chair and wore goggles, soft ear plugs and a headset that played electromagnetic noise. The subject put the shank of his dominant leg into the inflatable sleeve that was fixed to the testing arm of the isokinetic dynamometer device by straps. Initially, the subject was asked to alter the flexion angle of the knee joint from $90^{\circ}$ to $105^{\circ}, 120^{\circ}$, or $135^{\circ}$ actively and randomly, and all of these angles were used as the target angle. The subject was asked to maintain the angle for 5 seconds and to pay attention to the position of the joint. Then, the subject's knee joint was allowed to passively go back to the $90^{\circ}$ position. The subject moved passively and relied on his self-judgment to reset the former angle. Each angle was tested 3 times, and the angular velocity of passive movement was $5 \%$. The differences in the results between the two angles were compared. The precision of the test results was determined, and the absolute error of the results of the two angles was used an index of proprioception ${ }^{11)}$.

The motion minimum threshold measurement test: The subject sat on the testing chair and wore goggles, soft ear plugs and a headset that played electromagnetic noise. The subject put the shank of his dominant leg into the inflatable sleeve that was fixed to the testing arm of the isokinetic dynamometer device by straps. In the time period of 3-10 s after telling the subject the test had begun, the servo power device was used to provide a continuous, slow and passive joint movement at a random time. The angular velocity of passive movement was $0.5^{\circ} / \mathrm{s}$, and the starting flexion angles of the knee joint were $105^{\circ}, 120^{\circ}$ and $135^{\circ}$. The movement at each angle was tested in the clockwise direction 3 times and in the counter-clockwise direction 3 times. The motion threshold of passive movement that the joint could perceive, the difference between the joint angle at the start of the movement and the joint angle that the subject could perceive during the movement, was measured and compared. The precision of the test results was determined, and the absolute error of the results of the two angles was used an index of proprioception.

The force sense reproduction method: The subject sat on the testing chair, with the torso and thigh of the dominant side fixed by straps to eliminate muscle strength compensation of joints other than the tested knee joint. The axes of rotation of the knee joint and of the power testing were aligned. The isokinetic dynamometer device was set to the isometric muscle strength testing mode. First, the subject's maximal voluntary isometric contraction was tested. The flexion angles of the knee joint were $105^{\circ}, 120^{\circ}$ and $135^{\circ}$, and the tests were conducted in the direction of knee extension at each angle. The maximum voluntary isometric muscle contraction (MVIC) of muscle strength was obtained. At each joint angle, the isometric stretching muscle strength test was conducted for 5 seconds, followed by a rest of 5 seconds after each test, and a rest of 300 seconds after each group of tests to eliminate the fatigue effect. The test coefficient of variance (CV) was controlled below $15 \%$. After resting for 30 minutes and setting $50 \%$ of the MVIC as the target force value, the subject was asked to maintain the target

Table 1. General characteristics of the subjects $(\mathrm{N}=30)$

\begin{tabular}{lcc}
\hline Variables (unit) & Subjects & Mean \pm SD \\
\hline Gender (male/female) & $30 / 0$ & \\
Age (years) & & $19.3 \pm 1.0$ \\
Height (cm) & & $170.6 \pm 5.3$ \\
Weight (kg) & $63.0 \pm 12.1$ \\
\hline
\end{tabular}


force value. A line was drawn on the computer screen representing the target force value, and the subject controlled the force using visual feedback. Simultaneously, the subject was asked to pay attention to the range of force that he used. The subject was then asked to close his eyes and produce the same force output, recorded as the actual measured force value, and to maintain that force for 5 seconds. The differences between the target force values and the actual measured force values were calculated. The absolute error of the two values was used an index of proprioception ${ }^{12}$.

The statistical software SPSS20.0 was used for the statistical analysis of the test results. All of the test results are presented as the average value \pm the standard deviation. The corelations among the results of the joint angle reset method, the motion minimum threshold measurement method, and the force sense reproduce method were analysed using Pearson's correlation coefficient.

\section{RESULTS}

Table 2 shows that the result of the joint angle reset method was $7.49 \pm 4.94^{\circ}$; the result of the motion minimum threshold measurement method was $1.07 \pm 0.35^{\circ}$; and the result of the force sense reproduction method was $3.22 \pm 4.18 \mathrm{Nm}$.

Table 3 shows that the correlation coefficient between the joint angle reset method and the motion minimum threshold measurement method was 0.29 ; the correlation coefficient between the joint angle reset method and the force sense reproduce method was 0.41 ; and the correlation coefficient between the motion minimum threshold measurement method and the force sense reproduce method was 0.15 . All of the correlation coefficients were less than 0.6 . Therefore, no significant correlation was found among these three test results $(\mathrm{p}>0.05)$. The correlations in descending order from strong to weak are the correlation between the joint angle reset method and the force sense reproduction method, the correlation between the joint angle reset method and the motion minimum threshold measurement method, and the correlation between the motion minimum threshold measurement method and the force sense reproduction method.

\section{DISCUSSION}

At present, proprioception is defined as nerve impulses sent out by the mechanoreceptors of joints, joint capsules, ligaments, muscles, tendons and skin to the central nervous system ${ }^{13-15)}$. Proprioception is the perception of muscle tone that links movement and joint position and is usually divided into force sense, motion sense, and position sense. Proprioception represents the discriminating ability of joint muscle strength. Motion sense refers to the ability to identify joint movement, including the direction, amplitude, velocity, acceleration, time, et cetera of movement. Position sense refers to the ability to reproduce the given joint angle actively or passively. Three capabilities can function consciously or unconsciously to ensure the automatic control, balance and joint stability of human movement, which enables normal physical activities and activities of daily living ${ }^{16)}$.

The joint angle reset method mainly reflects position sense; the motion minimum threshold measurement method mainly reflects motion sense; and the force sense reproduction method mainly reflects force sense. Each test method reflects only one aspect of proprioception. Therefore, using the results obtained from using only one test method as an index of proprioception is one-sided.

Table 2. Results of the joint angle reset method, the motion minimum threshold measurement method, and the force sense reproduction method

\begin{tabular}{lccc}
\hline & $\begin{array}{c}\text { Joint angle reset method } \\
\left({ }^{\circ}\right)\end{array}$ & $\begin{array}{c}\text { Motion minimum threshold } \\
\text { measurement method }\left({ }^{\circ}\right)\end{array}$ & $\begin{array}{c}\text { Force sense reproduction } \\
\text { method (Nm) }\end{array}$ \\
\hline Maximum & 18.83 & 3.03 & 9.02 \\
Minimum & 0.87 & 0.63 & 0.12 \\
Average value & 7.49 & 1.07 & 3.22 \\
Standard deviation & 4.94 & 0.35 & 4.18 \\
\hline
\end{tabular}

Table 3. Correlation of the results of the joint angle reset method, the motion minimum threshold measurement method, and the force sense reproduction method

\begin{tabular}{lccc}
\hline & $\begin{array}{c}\text { Joint angle reset } \\
\text { method }\end{array}$ & $\begin{array}{c}\text { Motion minimum threshold } \\
\text { measurement method }\end{array}$ & $\begin{array}{c}\text { Fonse reproduction } \\
\text { method }\end{array}$ \\
\hline Joint angle reset method & 1 & 0.29 & 0.41 \\
Motion minimum threshold measurement method & 0.29 & 1 & 0.15 \\
Force sense reproduction method & 0.41 & 0.15 & 1 \\
\hline
\end{tabular}


No significant correlation was found among the results obtained using the joint angle reset method, the motion minimum threshold measurement method, and the force sense reproduce method, which indicates that there is no correlation among the position sense, motion sense, and force sense that they respectively reflect.

Proprioceptive information is generated by the mechanoreceptors of joints, joint capsules, ligaments, muscles, tendons and skin. The main receptors that generate proprioceptive information are located in the muscles, tendons, ligaments and joint capsules, whereas those that are located in the deeper layers of the skin and fascia are traditionally viewed as complementary sources. Mechanoreceptors transform the mechanical change of the deformation of human tissue into nerve signals and send them to the central nervous system through the sensory pathways ${ }^{17}$. The importance of different mechanoreceptor functions in proprioception is the focus of academic debate. The current view is that proprioception is mainly composed of the receptor signalling of muscle spindles and that the feeling of tension is provided by the muscle receptors called Golgi tendon organs ${ }^{18)}$. Joint receptors play only a small role in proprioception in the middle range of joint motion, and they are only fully activated at the extreme positions of both sides of the range of motion. Like joint receptors, skin receptors are also fully activated only at the extreme positions of both sides of the range of motion ${ }^{19}$ ). In contrast, the muscle spindle is unanimously considered to be activated throughout the whole range of motion and can provide proprioceptive information ${ }^{20)}$. In short, the muscle receptors, especially muscle spindles, are the main proprioceptors. The other proprioceptors, including joints and skin receptors, only work at the extreme positions of joint motion, which seem to play a defining role in the range of joint motion ${ }^{21,22)}$.

This study tested knee joint angles of $105^{\circ}, 120^{\circ}$, and $135^{\circ}$ in the middle of the range of joint motion. In this range, the joint receptors and skin receptors are not sufficiently activated and contribute little to proprioception. The muscle spindles and Golgi tendon organs do most of the work. The main difference in the three types of test used is whether the muscles are involved. In the force sensation test, a large number of muscles perform isometric contraction, which may depend much more on the sensory input information of Golgi tendon organs. In the process of the motion sense test, the test joint is always in passive motion, so there is no muscle involvement, which depends much more on the input information of muscle spindles. In the position sense test, the gravitational force of the motion segment itself and the test arm need to be overcome, so muscle strength is involved. Moreover, during the test, when the joint angle changes, the muscle spindles are involved. Therefore, the position sense test relies on the double input of information from the muscle spindles and the Golgi tendon organs. The test results show that the correlations in descending order from strong to weak are the correlation between the joint angle reset method and the force sense reproduction method, the correlation between the joint angle reset method and the motion minimum threshold measurement method, and the correlation between the motion minimum threshold measurement method and the force sense reproduction method. The correlation between position sense, which mainly depends on the double input information of muscle spindles and Golgi tendon organs, and the other two senses (motion sense and force sense) was higher than the correlation between motion sense and force sense that mainly depend on either the muscle spindles or Golgi tendon organs, which confirms the previous conclusion.

No correlation was found among the results obtained using the joint angle reset method, the motion minimum threshold measurement method, and the force sense reproduction method. Therefore, no correlation was found among the position sense, the motion sense, and the force sense represented by each of these methods. Using the results obtained from using only one of the test methods, namely, the joint angle reset method, the motion minimum threshold measurement method, or the force sense reproduction method, as an index of proprioception is one-sided.

Force sense depends more on the sensory input information of Golgi tendon organs, motion sense depends more on the input information of muscle spindles, and position sense relies on the double input information of muscle spindles and Golgi tendon organs.

\section{ACKNOWLEDGEMENT}

This work was funded by the Ministry of Education of Humanities and Social Science project (11YJA880037), Beijing Normal University Teaching Construction and Reform Project (12-01-13).

\section{REFERENCES}

1) Sherrington C: The Integrative Action of the Nervous System. UK: Cambridge University Press, $1906: 74$.

2) Choi YK, Nam CW, Lee JH, et al.: The effects of taping prior to PNF treatment on lower extremity proprioception of hemiplegic patients. J Phys Ther Sci, 2013, 25: 1119-1122. [Medline] [CrossRef]

3) Ko TS, Lee SM, Lee DJ: Manual therapy and exercise for OA knee: effects on muscle strength, proprioception, and functional performance. J Phys Ther Sci, 2009, 21: 293-299. [CrossRef]

4) Ribeiro F, Oliveira J: Effect of physical exercise and age on knee joint position sense. Arch Gerontol Geriatr, 2010, 51: 64-67. [Medline] [CrossRef]

5) Ferreira LA, Santos LC, Pereira WM, et al.: Analysis of thoracic spine thrust manipulation for reducing neck pain. J 
Phys Ther Sci, 2013, 25: 325-329. [CrossRef]

6) Riemann BL, Lephart SM: The sensorimotor system, part I: the physiologic basis of functional joint stability. J Athl Train, 2002, 37: 71-79. [Medline]

7) Song BK, Chung SM, Hwang BY: The effects of somatosensory training focused on the hand on hand function, postural control and ADL of stroke patients with unilateral spatial neglect and sensorimotor deficits. J Phys Ther Sci, 2013, 25: 297-300. [CrossRef]

8) Barrett DS: Proprioception and function after anterior cruciate reconstruction. J Bone Joint Surg Br, 1991, 73: 833-837. [Medline]

9) Ko T, Jeong U, Lee K: Effects of the inclusion thoracic mobilization into cranio-cervical flexor exercise in patients with chronic neck pain. J Phys Ther Sci, 2010, 22: 87-91. [CrossRef]

10) MacDonald PB, Hedden D, Pacin O, et al.: Proprioception in anterior cruciate ligament-deficient and reconstructed knees. Am J Sports Med, 1996, 24: 774-778. [Medline] [CrossRef]

11) Kim C-Y, Choi J-D, Kim H-D: No correlation between joint position sense and force sense for measuring ankle proprioception in subjects with healthy and functional ankle instability. Clin Biomech (Bristol, Avon), 2014, 3: 977-983.

12) Maenhout AG, Palmans T, De Muynck M: The impact of rotator cuff tendinopathy on proprioception, measuring force sensation. J Shoulder Elbow Surg, 2012, 3: 1080-1086.

13) Carpenter JE, Blasier RB, Pellizzon GG: The effects of muscle fatigue on shoulder joint position sense. Am J Sports Med, 1998, 26: 262-265. [Medline]

14) Ribeiro F, Mota J, Oliveira J: Effect of exercise-induced fatigue on position sense of the knee in the elderly. Eur J Appl Physiol, 2007, 99: 379-385. [Medline] [CrossRef]

15) Voight ML, Hardin JA, Blackburn TA, et al.: The effects of muscle fatigue on and the relationship of arm dominance to shoulder proprioception. J Orthop Sports Phys Ther, 1996, 23: 348-352. [Medline] [CrossRef]

16) Miura K, Ishibashi $Y$, Tsuda E, et al.: The effect of local and general fatigue on knee proprioception. Arthroscopy, 2004, 20: 414-418. [Medline] [CrossRef]

17) Grigg P: Peripheral neural mechanism in proprioception. J Sport Rehabil, 1994, 3: 2-17.

18) Proske U: Kinesthesia: the role of muscle receptors. Muscle Nerve, 2006, 34: 545-558. [Medline] [CrossRef]

19) Burke D, Gandevia SC, Macefield G: Responses to passive movement of receptors in joint, skin and muscle of the human hand. J Physiol, 1988, 402: 347-361. [Medline] [CrossRef]

20) Macefield G, Gandevia SC, Burke D: Perceptual responses to microstimulation of single afferents innervating joints, muscles and skin of the human hand. J Physiol, 1990, 429: 113-129. [Medline] [CrossRef]

21) Goble DJ, Coxon JP, Wenderoth N, et al.: Proprioceptive sensibility in the elderly: degeneration, functional consequences and plastic-adaptive processes. Neurosci Biobehav Rev, 2009, 33: 271-278. [Medline] [CrossRef]

22) Proske U, Gandevia SC: The kinaesthetic senses. J Physiol, 2009, 587: 4139-4146. [Medline] [CrossRef] 